\title{
Histopathological and immunohistochemical investigations of the hepatic compartment associated with parasitism and serum biochemical changes in canine visceral leishmaniasis
}

\author{
Rodolfo Cordeiro Giunchetti ${ }^{\mathrm{a}, \mathrm{b}}$, Wilson Mayrink ${ }^{\mathrm{c}}$, Cláudia Martins Carneiro ${ }^{\mathrm{a}, \mathrm{d}}$, \\ Rodrigo Corrêa-Oliveira ${ }^{b}$, Olindo Assis Martins-Filho ${ }^{\mathrm{e}}$, Marcos José Marques ${ }^{\mathrm{c}}$, \\ Washington Luiz Tafuri ${ }^{\text {a }}$, Alexandre Barbosa Reis ${ }^{a, b, d, *}$

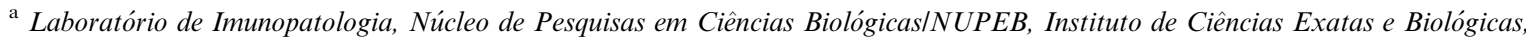 \\ Universidade Federal de Ouro Preto, Ouro Preto, MG, 400-000 CEP 35, Brasil \\ ${ }^{\mathrm{b}}$ Laboratório de Imunologia Celular e Molecular, Instituto de Pesquisas René Rachou, Fundação Oswaldo Cruz, Belo Horizonte, \\ Minas Gerais, Brasil ( $R C G, R C-O, A B R$ ) \\ ${ }^{\mathrm{c}}$ Laboratório de Leishmanioses, Departamento de Parasitologia, Instituto de Ciências Biológicas, Universidade Federal de Minas Gerais, \\ Belo Horizonte, Minas Gerais, Brasil \\ d Departamento de Análises Clínicas, Escola de Farmácia, Universidade Federal de Ouro Preto, Ouro Preto, Minas Gerais, Brasil

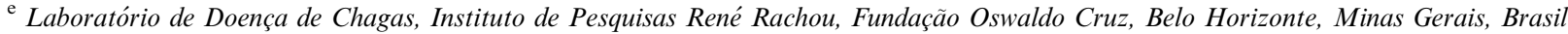

Accepted 26 April 2007

\begin{abstract}
The immunopathological evaluation of the hepatic compartment associated with parasitism and biochemical findings are essential for understanding the genesis of hepatomegaly in canine visceral leishmaniasis (CVL). Three clinical groups of dogs naturally infected with Leishmania chagasi [i.e., asymptomatic ( $\mathrm{AD}, n=12)$, oligosymptomatic (OD, $n=12)$ and symptomatic (SD, $n=17)$ ] were assessed and compared with a group of non-infected dogs (NID, $n=11)$. Intense reaction of the Kupffer cells, capsule and portal inflammation, and the presence of intralobular granulomas, were observed in the different clinical groups. Dogs in the SD group presented a higher frequency of parasitism compared with the AD group. Inflammatory alterations were more intense in the SD group and were associated with parasitism. Our results indicated an association between histological liver changes and the progression of biochemical alterations according to progression of clinical forms of CVL, and the direct relationship between clinical symptoms and frequency of hepatic parasitism.
\end{abstract}

(c) 2007 Elsevier Ltd. All rights reserved.

Keywords: Dog; Histopathology; Immunohistochemistry; LDU index; Leishmania chagasi; Liver; Parasitism; Visceral leishmaniasis

\section{Introduction}

Human visceral leishmaniasis (HVL), one of the most important zoonoses, is caused by the protozoa Leishmania

\footnotetext{
* Corresponding author. Address: Laboratório de Imunopatologia, Núcleo de Pesquisas em Ciências Biológicas/NUPEB, Instituto de Ciências Exatas e Biológicas, Universidade Federal de Ouro Preto, Ouro Preto, MG, 400-000 CEP 35, Brasil. Tel.: +55 313559 1694; fax: +55 31 35591680.

E-mail addresses: giunchetti@nupeb.ufop.br (R.C. Giunchetti), alexreis@nupeb.ufop.br (A.B. Reis).
}

(Leishmania) chagasi. Dogs are considered to be the main domestic reservoir of HVL since they host the amastigote forms of the parasite that are able to infect the insect vector (Abranches et al., 1991). Dogs are excellent models for the study of HVL because the natural history of the disease in dog and man is similar (Moreno and Alvar, 2002). Furthermore, infected dogs are frequently presented as potential patients in small-animal clinics in endemic areas. We have recently shown that the clinical evolution of canine visceral leishmaniasis (CVL) in naturally infected dogs involves clear alterations in serological, parasitological and 
biochemical/haematological parameters, and that such alterations are directly correlated with the clinical status of the disease (Giunchetti et al., 2006; Reis et al., 2006).

In one of the first histological studies (Meleney, 1925), outlined the relationship between hepatic parasitism and progressive alterations in the parenchyma of the liver. More recently, Andrade and Andrade (1966) described the dilation of the venous sinusoids and swelling of the hepatocytes and Kupffer cells frequently parasitised. These authors also reported the diffuse infiltration of plasma lymphocytes into the parenchyma with the formation of sparse granulomas. Similarly, experimental dogs that had been infected with $L$. $(L$.) infantum exhibited moderate hepatitis characterised by periportal infiltration of the lymphoplasmacytic cells, as well as macrophages, often infected by amastigote forms of the parasite (Binhazim et al., 1993). Dogs naturally infected with $L$. (L.) chagasi, additionally presented intralobular and intravascular granulomas in the liver, consisting predominantly of parasitised and non-parasitised macrophages, epithelioid cells, and a small number of lymphocytes together with rare granulocytes (neutrophils), (Tafuri et al., 1996). Despite the high frequency of amastigote forms found inside the hypertrophic and hyperplastic Kupffer cells, these authors did not establish a relationship between parasitism and the number of such cells or the size of the granulomas.

We have recently demonstrated that parasite density in the skin, bone marrow and spleen compartments, increased according to the severity of clinical manifestations (Giunchetti et al., 2006; Reis et al., 2006). Using indirect immunofluorescence assays, it was possible to demonstrate that symptomatic dogs displayed higher serological titres compared with asymptomatic dogs, and that the severity of the disease was correlated with total IgG titres. In addition, symptomatic dogs presented lower albumin/globulin (A/G) ratios compared with asymptomatic and control dogs. These data suggested a relationship between the presence of exacerbated clinical forms of CVL, serum protein levels and humoral immune response alterations.

Although the histological alterations that occur in the liver during VL are well reported, the association between the intensity of such lesions and the parasitic load has not been established. In the present study, potential correlations between the histological/parasitological modifications of the liver and biochemical changes in the blood serum, including albumin, $\mathrm{A} / \mathrm{G}$ ratio, total protein and plasma globulin levels, have been investigated. Knowledge of such correlations would be of value in elucidating the involvement of the liver in CVL and the systemic consequences.

\section{Materials and methods}

The details of the proposed study were presented to, and approved by, the Ethical Committee for the Use of Experimental Animals of the Universidade Federal de Minas Gerais (CETEA).

\subsection{Animal population}

Fifty-two adult dogs aged between 2 and 6 years, of both genders and of mixed breed, were supplied by the Centro de Controle de Zoonoses of Belo Horizonte, State of Minas Gerais, Brazil. The animals were maintained in kennels at the Leishmaniasis Laboratory of the Parasitology Department, Institute of Biological Sciences, Universidade Federal de Minas Gerais, Belo Horizonte, Brazil. Dogs were subjected to serological anti-Leishmania IgG assessment conducted using the indirect immunofluorescence antibody test (IFAT) following the instructions provided with the commercial kit supplied by Biomanguinhos (Fiocruz, Rio de Janeiro, RJ, Brazil). Eleven animals presented parasitological and serological tests that were negative for Leishmania (antibody titres lower than 1:40), and these non-infected dogs (NID) were regarded as control animals. Forty-one dogs were found to have been naturally infected with $L$. ( $L$. ) chagasi according to the parasitological and serological tests. VL-positive animals were classified clinically according to the presence or absence of signs of infection as follows: asymptomatic dogs (AD; $n=12$ ) showed no signs indicative of the disease, oligosymptomatic dogs (OD; $n=12)$ exhibited a maximum of three clinical signs, whilst symptomatic dogs $(\mathrm{SD} ; n=17)$ exhibited more than three clinical signs (Mancianti et al., 1988; Reis et al., 2006).

\subsection{Collection of liver samples and histological examinations}

Euthanasia was performed by intravenous overdose of barbiturate. Liver samples were collected, stored in $10 \%$ neutral buffered formalin and then submitted to routine histological procedures [following staining with haematoxylin-eosin (HE)] or to anti-Leishmania immunohistochemical examination.

Inflammation of the hepatic capsule and the portal tract as well as the presence of intralobular granulomas and hypertrophic/hyperplastic Kupffer cells, were assessed histologically by examination of the HE-stained liver sections under an optical microscope. The histological alterations were graded according to the degree of damage as follow: - , no histological alteration; +, isolated foci of inflammation, less than 2 intralobular granulomas and 5 Kupffer cells per field at $\times 400$ magnification; ++ , isolated or coalescing areas of histological alteration including inflammation, 2-4 intralobular granulomas and 5-10 Kupffer cells per field at $\times 400$ magnification; +++ , diffuse areas of histological alteration including inflammation, more than 4 intralobular granulomas and 10 Kupffer cells per field at $\times 400$ magnification.

\subsection{Parasitological examination}

Parasitological diagnoses in liver smears were performed after necropsy of the animals. The smears were stained by Giemsa and examined by optical microscopy for the identi- 
fication of amastigote forms of Leishmania. Parasite density was determined and expressed using the Leishman-Donovan unit (LDU) index defined to be the number of Leishmania amastigotes per 1000 nucleated cells (Stauber, 1956).

The anti-Leishmania immunohistochemical assay was also used to investigate parasite density (Tafuri et al., 2004). Semi-quantitative analysis was carried out under the optical microscope in order to detect the immunolabelled amastigote forms and to establish the degree of parasitism. The parasite load in the liver sections was determined according to Giunchetti et al. (2006) as the average number of micro-organisms identified in five optical fields (at $\times 400$ magnification) and classified as: - , no microorganisms; + , light density $(1-100)$; ++ , moderate density (101-300); and +++ , intense parasitism $(>300)$.

\subsection{Biochemical evaluation of serum}

Total serum proteins were determined by the Biuret method, with spectrophotometric readings being taken at $510 \mathrm{~nm}$ on a Celm (Barueri-SP, Brazil) E-225D spectrophotometer. The electrophoretic profile of the serum proteins (plasma albumin and globulin) was established using Celogel ${ }^{\circledR}$ (Chemetron, Milan, Italy) cellulose acetate strips according to the manufacturer's instructions. Serum proteins were identified spectrophotometrically using CS93101 PC software.

\subsection{Statistical analysis}

Statistical analyses were performed using the Prism 3.0 software package (GraphPad, San Diego, CA, USA). Between-group comparisons of the results obtained from the anti-Leishmania immunohistochemical, biochemical evaluation of serum and histopathological assays were performed using the Kruskal-Wallis test. Analysis of the LDU index was carried out using the $\chi^{2}$ test, whilst possible correlations between the different approaches were established with the Spearman test. Differences were considered significant when the probabilities of equality ( $P$ values) were $\leqslant 0.05$.

\section{Results}

\subsection{Macroscopical and histological evaluation}

During the necropsy proceedings we observed hyperaemia/congestion in naturally infected dogs with $L$. $(L$.) chagasi and liver enlargement characterizing hepatomegaly directly related to the severity of clinical disease (Table 1).

The main histological findings observed in the liver samples derived from dogs naturally infected with $L$. $(L$.) chagasi are presented in Fig. 1a-e (HE stain) and in $\mathrm{f}$ (immunohistochemical reaction). Severe inflammation of the hepatic capsule with infiltration of mononucleated cells, especially plasmocytes, could be readily observed (Fig. 1a). Fig. 1b shows the intense infiltration of inflammatory cells in the portal tract, especially mononucleated plasmocytes, histiocytes and lymphocytes. Intralobular granulomas were dispersed throughout the hepatic parenchyma (Fig. 1c and d), whilst hypertrophy/hyperplasia of the Kupffer cells was clearly visible (Fig. 1e).

The frequency of inflammation, as determined by the number of inflammatory cells in the hepatic capsule and the portal tract, and the number of intralobular granulomas and hypertrophic/hyperplastic Kupffer cells, was significantly higher $(P<0.05)$ in dogs naturally infected with $L$. (L.) chagasi (groups $\mathrm{AD}, \mathrm{OD}$ and $\mathrm{SD}$ ) compared with the NID group (Table 1). Additionally, the SD group exhibited a higher frequency $(P<0.05)$ of hypertrophic/ hyperplastic Kupffer cells compared with the AD and OD groups (Fig. 1e; Table 1).

The semi-quantitative analyses of capsule inflammation in OD showed higher $(P<0.05)$ intensity than NID group (Fig. 2a). The SD group presented higher $(P<0.05)$ intensity of inflammation in the portal tract in relation to NID group (Fig. 2b). The naturally infected dogs (AD, OD and SD) presented an increase $(P<0.05)$ in the intensity of intralobular granulomas when compared to NID (Fig. 2c). Furthermore, the SD group showed an increase $(P<0.05)$ in the intensity of hypertrophic/hyperplastic Kupffer cells when compared to NID group (Fig. 2d).

Table 1

Histopathological and parasitological evaluation of the liver of CVL infected and non-infected dogs

\begin{tabular}{|c|c|c|c|c|}
\hline Parameters & NID $(n=11)$ & $\mathrm{AD}(n=12)$ & $\mathrm{OD}(n=12)$ & $\mathrm{SD}(n=17)$ \\
\hline \multicolumn{5}{|l|}{ Number (and \%) of dogs with hepatic changes } \\
\hline Hepatomegaly & 0 & $1(8,3)$ & $8(66,7)^{\S, \oplus}$ & $16(94,1)^{\S, \uparrow}$ \\
\hline Capsule inflammation & 0 & $5(41.7)^{\S}$ & $8(66.7)^{\S}$ & $8(47.1)^{\S}$ \\
\hline Portal inflammation & $3(27.3)$ & $9(75.0)^{\S}$ & $9(75.0)^{\S}$ & $16(94.1)^{\S}$ \\
\hline Presence of intralobular granuloma & $1(9.1)$ & $9(75.0)^{\S}$ & $9(75.0)^{\S}$ & $12(70.6)^{\S}$ \\
\hline Hypertrophy/Hyperplasia of Küpffer cells & $1(9.1)$ & $6(50.0)^{\S}$ & $6(50.0)^{\S}$ & $15(88.2)^{\top, \S}$ \\
\hline Anti-Leishmania immunohistochemical assay & 0 & $3(25.0)^{\S}$ & $6(50.0)^{\S}$ & $11(64.7)^{\top, \S}$ \\
\hline Parasitological diagnosis & 0 & $6(50.0)^{\S}$ & $7^{*}(63.6)^{\S}$ & $15^{\|}(93.8)^{\top, \S}$ \\
\hline \multicolumn{5}{|l|}{ Mean value \pm standard deviation } \\
\hline LDU index & - & $72.5 \pm 162.4$ & $49.5 \pm 79.4^{*}$ & $268 \pm 593.5^{\|}$ \\
\hline
\end{tabular}

NID, non-infected dogs; AD, asymptomatic dogs; OD, oligosymptomatic dogs; SD, symptomatic dogs; $n$, number of animals in each group; parasitological diagnosis $=$ the number of dogs identified as infected according to their LDU index; ${ }^{*} n=11 ;{ }^{n} n=16$. Significant differences $(P<0.05)$ are represented by the label $\S$ and $\uparrow$ related to the groups NID and AD, respectively. 

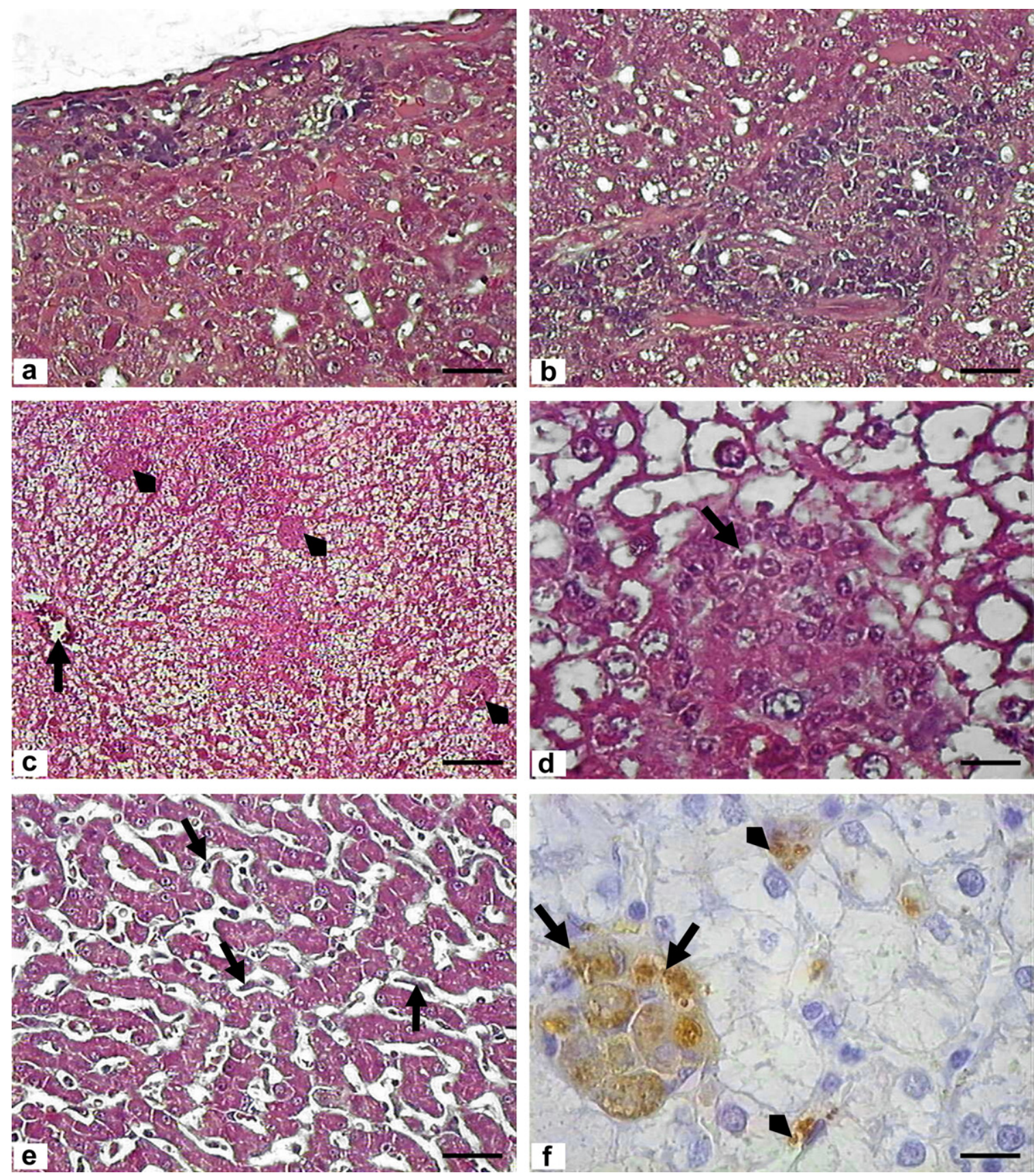

Fig. 1. (a) Liver, oligosymptomatic dog. Inflammation of the hepatic capsule showing capsule thickened and colonized by a chronic inflammatory reaction. HE. Bar $=25 \mu \mathrm{m}$. (b) Liver; symptomatic dog. Inflammation of portal tract showing cellular mononuclear infiltrate characterized by plasmocytes, macrophages and lymphocytes. HE. Bar $=25 \mu \mathrm{m}$. (c) Liver, symptomatic dog. Granulomatous inflammatory reaction with three intralobular granulomas dispersed in the hepatic parenchyma (arrow heads) and centrilobular vein (arrow). HE. Bar $=50 \mu \mathrm{m}$. (d) Liver, oligosymptomatic dog. Intralobular granuloma formation consisting of mononucleated cells, predominantly macrophages, one of which presents an amastigote parasite inside (arrowed). HE. Bar $=12,5 \mu \mathrm{m}$. (e) Liver, symptomatic dog. Intense hypertrophy/hyperplasia of the Kupffer cells (arrowed). HE. Bar $=25 \mu \mathrm{m}$. (f) Liver, symptomatic dog. Amastigotes parasites inside mononucleated cells of the granuloma (arrowed) and inside the Kupffer cells (arrow heads). AntiLeishmania immunohistochemistry. Bar $=12.5 \mu \mathrm{m}$.

\subsection{Parasitological evaluation}

The SD group exhibited a higher frequency $(P<0.05)$ of parasites than the AD group as revealed by the antiLeishmania immunohistochemical reaction. This observation was confirmed from a consideration of the parasitological diagnosis which showed that the frequency of parasites in the SD group was significantly higher $(P<0.05)$ than in the AD group (Table 1$)$. However, with respect to parasitic load, there were no significant differences in the mean values of the LDU index between the groups $\mathrm{AD}, \mathrm{OD}$ and $\mathrm{SD}$ (Table 1). Additionally, SD group shown higher intensity $(P<0.05)$ of the parasite burden as determined by anti-Leishmania immuno- 

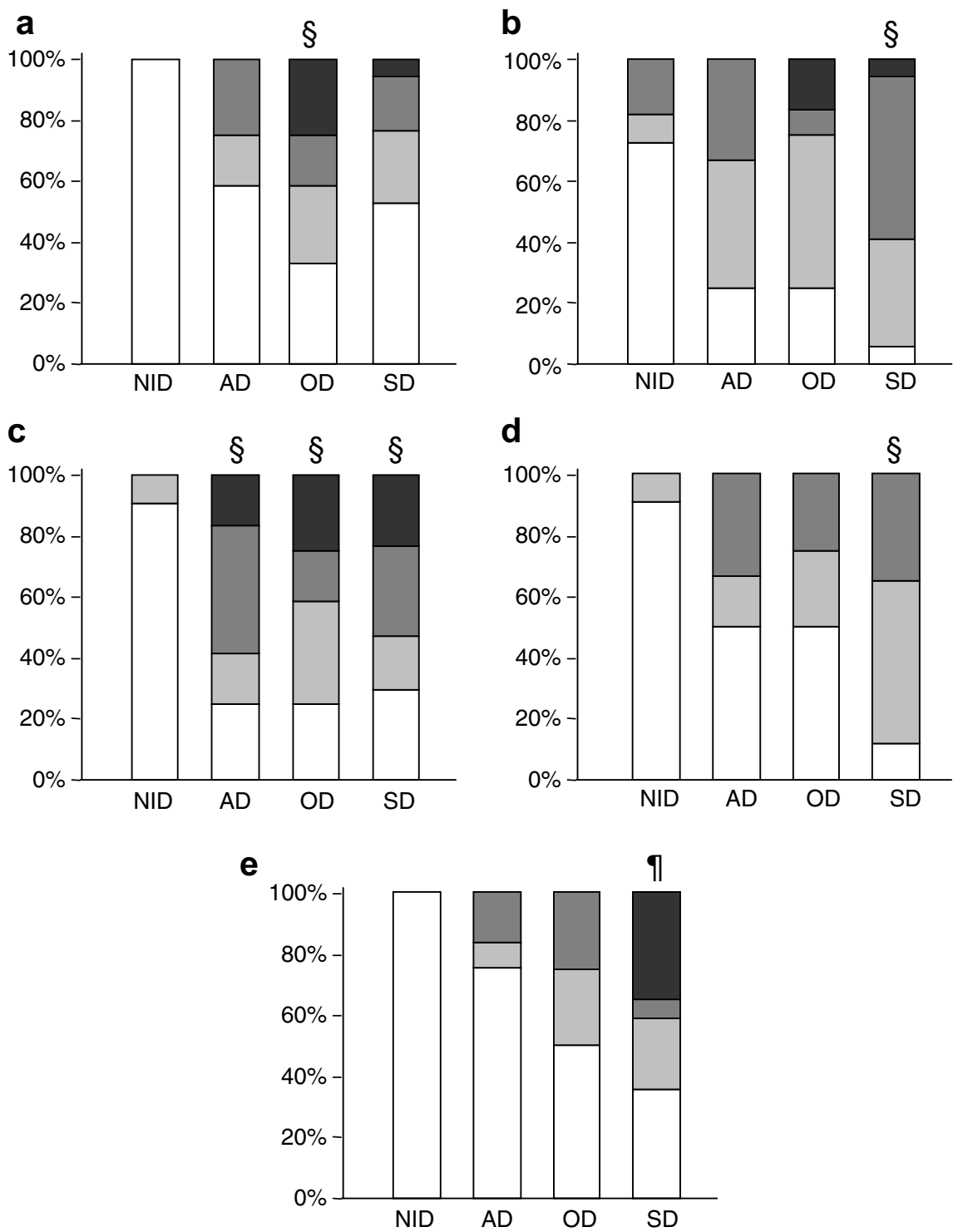

Fig. 2. Liver, naturally infected dogs with $L$. (L.) chagasi and non-infected dogs showing histological alterations in the groups: AD, asymptomatic; OD, oligosymptomatic; SD, symptomatic and NID, non-infected dogs. (a) Intensity of capsule inflammation. (b) Intensity of inflammation in portal tract. (c) Intensity of intralobular granulomas. (d) Intensity of hypertrophic/hyperplastic Kupffer cells. (e) Intensity of the parasitic burden as determined by anti-Leishmania immunohistochemical reaction. The bars are coded as follows: white $(-$, absence; light grey $(+)$, mild; dark grey $(++)$, moderate; black $(+++)$, intense. Significant differences $(P<0.05)$ between AD, OD and SD groups in relation to the NID group are represented by the label $\S$. Significant differences $(P<0.05) \mathrm{SD}$ in relation AD groups are represented by the label $\uparrow$.

histochemical reaction when compared to AD group (Fig. 2e).

\subsection{Biochemical evaluation of serum}

The biochemical evaluation of serum in the AD, OD and SD groups presented significant decrease $(P<0.05)$ in serum albumin compared to NID. Moreover, SD group showed significant reduction in serum albumin in relation to $\mathrm{AD}$ and $\mathrm{OD}$ groups (Fig. 3a). SD and OD groups showed higher levels $(P<0.05)$ in plasma globulin in relation to NID. Furthermore, SD group presented significant increase $(P<0.05)$ in plasma globulin compared to $\mathrm{AD}$ group (Fig. 3b). The evaluation in $\mathrm{A} / \mathrm{G}$ ratio showed signif- icant decrease $(P<0.05)$ in AD, OD and SD groups compared to NID. Moreover, SD group presented lower A/G ratios $(P<0.05)$ than AD group (Fig. 3c).

\subsection{Correlation between histologicallparasitological and biochemical results}

The correlations established between the results obtained using the histological/parasitological approach and the biochemical approaches are presented in Fig. $4 \mathrm{a}-$ h $(P<0.05)$. Hypertrophy/hyperplasia of the Kupffer cells was positively correlated with plasma globulin in the $\mathrm{AD}$ (Fig. 4a) and OD (Fig. 4c) groups, and with total serum protein content in the OD group (Fig. 4b). In the latter 

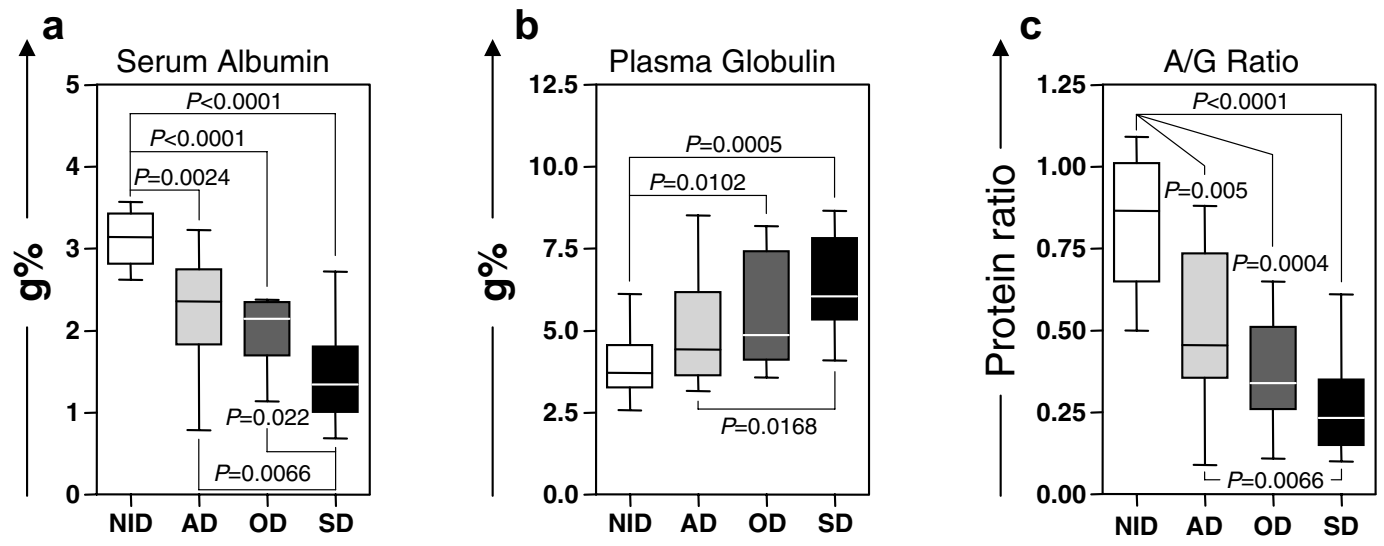

Clinical groups

Fig. 3. Evaluation of biochemical parameters of naturally infected (AD, OD and SD) and non-infected dogs (NID). The results are shown as average values \pm standard deviation. NID (white box); AD (clear grey box); OD (dark grey box) and SD (black box). Differences statistically significant were showed by connect lines between different groups. Serum albumin (a), plasma globulin (b) and A/G ratio (c) are shown.

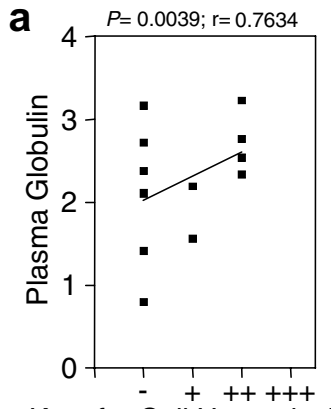

Kupper Cell Hyperplasia

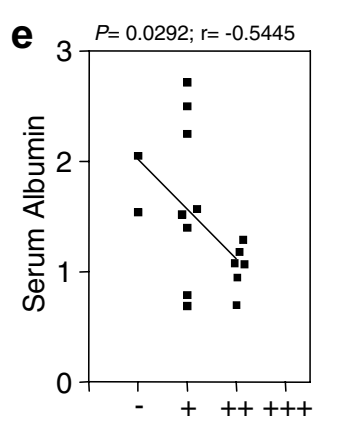

Kuppfer Cell Hyperplasia

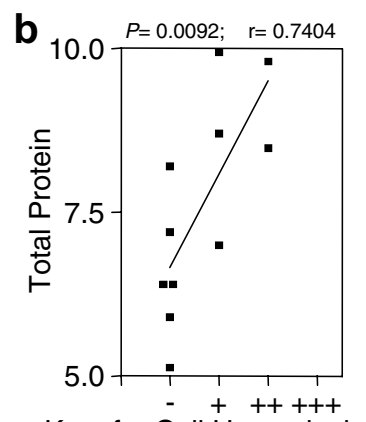

Kupper Cell Hyperplasia

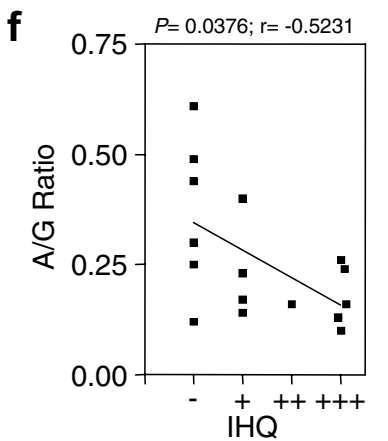

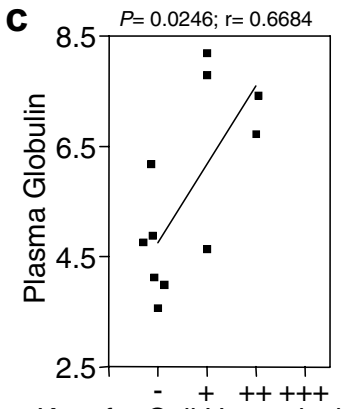

Kuppfer Cell Hyperplasia

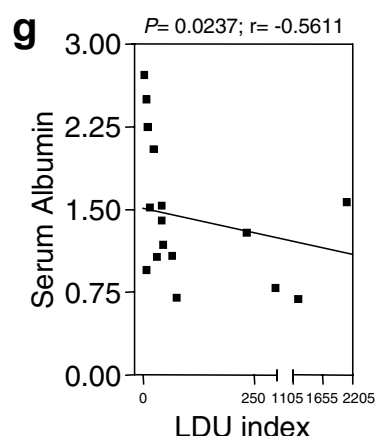

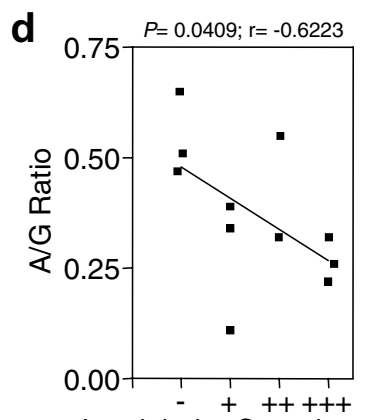

Intralobular Granuloma

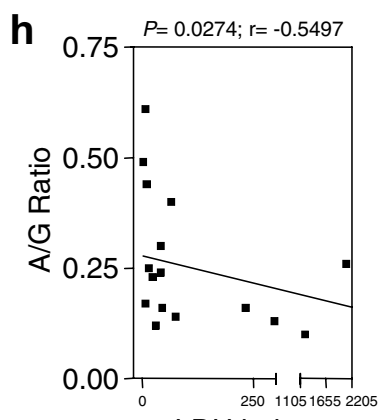

LDU index

Fig. 4. Liver, different clinical status of naturally infected dogs with L. (L.) chagasi. Correlation between the histological/parasitological examination and the biochemical serum evaluation. (a) Hypertrophy/hyperplasia of the Kupffer cells positively correlated with plasma globulin in the AD group. (b) Hypertrophy/hyperplasia of the Kupffer cells positively correlated with total serum protein content in the OD group. (c) Hypertrophy/hyperplasia of the Kupffer cells positively correlated with plasma globulin in the OD group. (d) Negative correlation between the albumin/globulin (A/G) ratio and the number of intralobular granulomas in OD group. (e) Negative correlations between serum albumin and hypertrophy/hyperplasia of Kuppfer cells in SD group. (f) Negative correlations between A/G ratio and immunohistochemical assay in SD group. (g) Negative correlations between serum albumin and LDU index in SD group. (h) Negative correlations between A/G ratio and LDU index. The results are expressed as scatter plots of individual measured values. Spearman correlation indexes $(r)$ at $P<0.05$ are shown, and connecting lines illustrate positive and negative correlation indexes.

group, there was a negative correlation between the albu$\mathrm{min} /$ globulin $(\mathrm{A} / \mathrm{G})$ ratio and the number of intralobular granulomas (Fig. 4d). The SD group showed negative correlations between all histological and biochemical parameters: thus the $\mathrm{A} / \mathrm{G}$ ratio was negatively correlated with the parasitological results (both immunohistochemical assay and LDU index, Fig. 4f and h, respectively), whilst serum albumin showed a negative correlation with both hypertrophy/hyperplasia of Kuppfer cells (Fig. 4e) and LDU index (Fig. 4g). 


\subsection{Analysis of the association between histological and parasitological parameters}

Relationships between the histological parameters were also investigated in $\mathrm{AD}, \mathrm{OD}$ and $\mathrm{SD}$ groups of naturally infected dogs in order to identify possible associations connected to the inflammatory reaction within the SD group. The parameters that presented significant differences $(P<0.05)$ between groups were: inflammation of the hepatic capsule, portal inflammation, and hypertrophy/hyperplasia of the Kupffer cells.

The associations between histological alterations and parasitism were also investigated in an attempt to understand their possible relationship. The anti-Leishmania immunohistochemical reaction allowed the establishment of significant associations $(P<0.05)$ between parasitism, portal inflammation and intralobular granulomas within group $\mathrm{AD}$ and between parasitism and portal inflammation within the SD group.

\section{Discussion}

Although histological alterations in the liver of murine animal models, men and dogs suffering from VL have been reported, it is very important to evaluate the intensity of such alterations and their relationships in order to understand the pathology of VL. CVL provides an excellent model for studying HVL according to Nieto et al. (1999) and was thus employed in the present study in order to evaluate biochemical parameters and correlations between the histological and biochemical alterations that occur during the progress of the disease.

Histological modifications of the liver in murine animals and men affected by VL typically relate to the presence of portal inflammation, comprising mainly mononucleated cells and hypertrophic/hyperplastic Kupffer cells (many of which are parasitised), and to the occurrence of granulomas, also consisting of mononucleated cells (Andrade and Andrade, 1966; Duarte and Corbett, 1987; El Hag et al., 1994; Gomes-Pereira et al., 2004; Gutierrez et al., 1984; Mangoud et al., 1997 and Murray, 2001). Similar alterations have also been observed in dogs affected by CVL (Binhazim et al., 1993; Tafuri et al., 1996; Tafuri et al., 2001) and are confirmed in the present study. The observed plasmocyte/histiocyte/lymphocyte infiltration of liver parenchyma, particularly mononucleated plasmocytes, reflects the polyclonal activation of the B cells characterising VL (Bourdoiseau et al., 1997; Duarte and Corbett, 1987; Gutierrez et al., 1984; Mangoud et al., 1997; Tafuri et al., 1996; Tafuri et al., 2001 and Tryphonas et al., 1997).

In the present study, all of the clinical groups of dogs infected with $L$. (L.) chagasi, i.e., $\mathrm{AD}, \mathrm{OD}$ and $\mathrm{SD}$, presented higher frequencies $(P<0.05)$ of both portal and of capsule inflammation compared with the NID group. Whilst inflammation of the hepatic capsule in CVL has not been the previous focus of attention, it is clearly of importance in the development of the histopathological events leading to CVL. Furthermore, the intense portal inflammation exhibited by the OD group compared with the NID group reinforces the implied relationship between such inflammation and the serious clinical consequences of the disease. Whilst it is clear that these inflammatory processes are stimulated by parasitism, they are not sufficient to eradicate the infection (Marquez and Martinez, 2001).

The presence of intralobular granulomas is one of the most important histopathological alterations found in HVL (Gutierrez et al., 1984; Mangoud et al., 1997 and Tafuri et al., 1996). In CVL, such granulomas are formed by epithelioid cells, a small number of lymphocytes and plasmocytes, and rare granulocytes, possibly containing amastigote forms inside the macrophages (Binhazim et al., 1993; Tafuri et al., 1996). The frequency of such intralobular granulomas within all clinical groups of $L$. $(L$.) chagasi-infected dogs was much higher than in the NID group, probably because the organism reacts to parasitism by sequestering the parasite in an attempt to control the multiplication of the amastigotes. An increase in the number of granulomas associated with the increase in parasite load in the liver has been demonstrated previously (Gutierrez et al., 1984 and Mangoud et al., 1997). The fact that granulomas are formed by inflammatory cells may facilitate their elimination from liver parenchyma after appropriate treatment. Moreover, evaluation of the granulomatous response in CVL may assist in the evaluation of liver tissue regeneration following experimental chemotherapy.

VL is characterised by accentuated hypertrophy/hyperplasia of the Kupffer cells (El Hag et al., 1994), the intensity of which depends on the degree of parasitism, as the organism attempts to restrain the multiplication of amastigotes (Mangoud et al., 1997; Tafuri et al., 1996; Tafuri et al., 2001; Tryphonas et al., 1997). This situation was readily observable in the SD group that also exhibited a higher frequency of these cell forms than did the AD or OD groups.

The intense capsule and portal inflammation associated with CVL may relationship to the hepatomegaly observed in the disease. Such tissue modifications could provide a mechanical barrier to blood flux and represent the histological basis for the enlargement of liver parenchyma. The present study reported a clear compromising in biochemical serum parameters directly related to the severity of clinical disease. In this context, SD that represent the extreme of clinical alterations presented significant increase in plasma globulin and decrease in serum albumin and A/G ratio compared to $\mathrm{AD}$ group. Thus, we confirm decreased levels of albumin and increased levels of globulin fraction, leading to inversion of $\mathrm{A} / \mathrm{G}$ ratio, according our previous study (Reis et al., 2006). Furthermore, in the present study, we have further shown that the progress of hypertrophy/ hyperplasia of the Kupffer cells was positively correlated with plasma globulin in the AD and OD groups and with total plasma protein in the OD group, but negatively correlated with plasma albumin concentration in the SD group. These data suggest that the Kupffer cells contribute 
to the alterations in protein content. Moreover, there was a negative correlation between the $\mathrm{A} / \mathrm{G}$ ratio and the number of intralobular granulomas within the OD group and with the immunohistochemical assay and the LDU index within the SD group. This finding implies that hepatic parasitism and consequential alterations, including the occurrence of intralobular granulomas, play a role in protein dysfunction that is commonly observed in OD and SD animals (Reis et al., 2006). A link is thus established between histopathological changes in the liver and progressive biochemical alterations in the blood.

In the present study, parasitological evaluation indicated that the frequency of hepatic parasitism in the SD group was higher than in the AD group (according to immunohistochemical assay and parasitological diagnosis). These findings imply a possible relationship between the intensity of hepatic parasitism and the evolution of clinical manifestations of CVL. Whilst studying the inflammation of the skin of the ears of dogs naturally infected with $L$. (L.) chagasi, Giunchetti et al. (2006) reported accentuated histological alterations and stronger parasitism in SD animals suggesting that cutaneous parasitism is accompanied by clinical manifestations of CVL. Furthermore, Reis et al. (2006) reported that, compared with SD animals, the bone marrow and spleen of AD dogs exhibited lower LDU indexes. Similarly, using the LDU index to compare L. (L.) chagasi-infected dogs, Sanchez et al. (2004) reported a more intense hepatic parasitism in SD than in AD animals. These authors suggested that the striking differences in parasite burden between AD and SD animals could be explained by a diminished infection or a more efficient control of the parasite replication in the former group, especially since the exact time of infection was unknown. Interestingly (Pinelli et al., 1994; Pinelli et al., 1995) had earlier proposed that SD animals may be more susceptible, or AD animals more resistant, towards infection by $L .(L$.) infantum.

Whilst studies of the association between histological alterations and hepatic parasitism are important for the understanding of CVL, the literature regarding such aspects is still limited. The present study has provided further evidence of the changes in SD animals between inflammatory events (hypertrophy/hyperplasia of the Kupffer cells, portal tract and intralobular granuloma). The higher frequency of parasitism within the SD group compared to the $\mathrm{AD}$ group was also established from the anti-Leishmania immunohistochemical assay and the parasitological diagnosis, since significant association $(P<0.05)$ between parasitism and portal inflammation was revealed in both groups. The presence of the parasite appears to be sufficient to induce an inflammatory reaction in the portal tract, although such reaction is not dependent on intensity or frequency of parasitism.

An association between parasitism and the presence of intralobular granulomas within the AD group of dogs was also detected in the present study, a relationship that has been reported by others (Binhazim et al., 1993;
Gutierrez et al., 1984; Mangoud et al., 1997). However, in agreement with previous findings, Tafuri et al. (1996) no relationship could be established between parasitism and the number/size of intralobular granulomas in the present work. This reinforces the hypothesis that the simple presence of the parasite in the hepatic parenchyma is sufficient to initiate events related to granulomatous inflammation. It may be that alterations in the hepatic tissue simply reflect the activation of the immune system. In support of this proposal, El Hag et al. (1994) observed that the inflammatory reaction persisted even after treatment and eradication of parasitism. On the basis of such evidence, we suggest that, along with parasitism, other factors are also involved in the inflammatory process.

\section{Acknowledgements}

This work was supported by Conselho Nacional de Desenvolvimento Cientifico e Tecnológico, Brazil (Grant No. 521124/98-0) and Fundação de Amparo à Pesquisa do Estado de Minas Gerais, Brazil (Grant No. CB0S 2222/ 97). The authors are grateful for the excellent technical assistance provided by Dr. Wagner Luiz Tafuri concerning the histopathological analyses. Finally, the authors are grateful for the network bioterism of Minas Gerais State.

\section{References}

Abranches, P., Silva-Pereira, M.C.D., Conceição-Silva, F., SantosGomes, G.M., Jans, J.G., 1991. Canine leishmaniasis: pathological and ecological factors influencing transmission of infection. J. Parasitol. 77, 561-577.

Andrade, Z.A., Andrade, S.G., 1966. Alguns novos aspectos da patologia do calazar (estudo morfológico de 13 casos necropsiados). Rev. Inst. Med. Trop. São Paulo 8, 259-266.

Binhazim, A.A., Chapman, W.L., Shin, S.S., Hanson, W.L., 1993. Determination of virulence and pathogenesis of a canine strain of Leishmania leishmania infantum in hamsters and dogs. Am. J. Vet. Res. 54, 113-121.

Bourdoiseau, G., Bonnefont, C., Magnol, J.P., Saint-Anré, I., Chabanne, L., 1997. Lymphocyte subset abnormalities in canine leishmaniasis. Vet. Immunol. Immunopathol. 56, 345-351.

Duarte, M.I.S., Corbett, C.E.P., 1987. Histopathological patterns of the liver involvement in visceral leishmaniasis. Rev. Inst. Med. Trop. São Paulo 29, 131-136.

El Hag, I.A., Hashim, F.A., El Toum, I.A., Homeida, M., El Califa, M., El Hassan, A.M., 1994. Liver morphology and function in visceral leishmaniasis (kala-azar). J. Clin. Pathol. 47, 547-551.

Giunchetti, R.C., Mayrink, W., Genaro, O., Carneiro, C.M., CoorêaOliveira, R., Martins-Filho, O.A., Marques, M.J., Tafuri, W.L., Reis, A.B., 2006. Influence of parasitism in dermal inflammatory focus in canine visceral leishmaniasis by Leishmania (Leishmania) chagasi. J. Comp. Pathol. 135, 100-107.

Gomes-Pereira, S., Rodrigues, O.R., Rolão, N., Almeida, P.D., SantosGomes, G.M., 2004. Hepatic cellular responses in mice with "cure" and "non-cure" phenotype to Leishmania infantum infection: importance of $\mathrm{CD}^{+} \mathrm{T}$ cells and TGF- $\beta$ production. FEMS Immunol. Med. Microbiol. 41, 59-68.

Gutierrez, Y., Maksem, J.A., Reiner, N.E., 1984. Pathologic changes in murine leishmaniasis (Leishmania donovani) with special reference to the dynamics of granuloma formation in the liver. Am. J. Pathol. 114, $222-230$. 
Mancianti, F., Gramiccia, M., Gradoni, L., Pieri, S., 1988. Studies on canine leishmaniasis control. I. Evolution of infection of different clinical forms in canine leishmaniasis following antimonils treatment. Trans. Roy. Soc. Trop. Med. Hyg. 82, 566-567.

Mangoud, A.M., Ramadan, M.E., Morsy, T.A., Amin, A.M., Mostafa, S.M., 1997. Histopathological studies of Syrian golden hamsters experimentally infected with Leishmania D. infantum. J. Egypt Soc. Parasitol. 27, 689-702.

Marquez, G., Martinez, A.C., 2001. Chemokine: the times are a changing. J. Clin. Invest. 107, 791-792.

Meleney, H.E., 1925. The histopathology of kala-azar in the hamster, monkey and man. Am. J. Pathol. 1, 147-168.

Moreno, J., Alvar, J., 2002. Canine leishmaniasis: epidemiological risk and experimental model. Trends Parasitol. 18, 399-405.

Murray, H.W., 2001. Tissue granuloma structure-function in experimental visceral leishmaniasis. Int. J. Exp. Pathol. 82, 249-267.

Nieto, C.G., García-Alonso, M., Requena, J.M., Mirón, C., Soto, M., Alonso, C., Navarrete, I., 1999. Analysis of the humoral immune response against total and recombinant antigens of Leishmania infantum: correlation with disease progression in canine experimental leishmaniasis. Vet. Immunol. Immunopathol. 67, 117-130

Pinelli, E., Killick-Kendrick, R., Wagenaar, J., Bernadina, W., Del Real, G., Ruitenberg, J., 1994. Cellular and humoral immune responses in dogs experimentally and naturally infected with Leishmania infantum. Infect. Immun. 1, 229-235.

Pinelli, E., Gonzalo, R.M., Boog, C.J.P., Rutten, V.P.M.G., Gebhard, D., Del Real, G., Ruitenberg, E.J., 1995. Leishmania infantum - specific T cell lines derived from asymptomatic dogs that lyse infected macro- phages in a major histocompatibility complex-restricted manner. Eur. J. Immunol. 25, 1594-1600.

Reis, A.B., Martins-Filho, O.A., Teixeira-Carvalho, A., Carvalho, M.G., Mayrink, W., França-Silva, J.C., Giunchetti, R.C., Genaro, O., Corrêa-Oliveira, R., 2006. Parasite density and impaired biochemi$\mathrm{cal} /$ hematological status are associated with severe clinical aspects of canine visceral leishmaniasis. Res. Vet. Sci. 81, 68-75.

Sanchez, M.A., Dias, N.L., Zerpa, O., Negron, E., Convit, J., Tapia, F.J., 2004. Organ-specific immunity in canine visceral leishmaniasis: analysis of symptomatic and asymptomatic dogs naturally infected with Leishmania chagasi. Am. J. Trop. Med. Hyg. 70, 618-624.

Stauber, L.A., 1956. Resistance to the Khartoum strain of Leishmania donovani. Rice Inst. Pamphlet 45, 80-96.

Tafuri, Wg.L., Oliveira, M.R., Melo, M.N., Tafuri, W.L., 2001. Canine visceral leishmaniosis: a remarkable histopathological picture of one case reported from Brazil. Vet. Parasitol. 96, 203-212.

Tafuri, Wg.L., Santos, R.L., Arantes, R.M.E., Gonçalves, R., Melo, M.N., Michalik, M.S.M., Tafuri, W.L., 2004. An alternative immunohistochemical method for detecting Leishmania amastigotes in paraffin-embedded canine tissues. J. Immunol. Methods 292, 17-23.

Tafuri, Wg.L., Tafuri, W.L., Barbosa, A.J.A., Michalick, M.S.M., Genaro, O., França-Silva, J.C., Mayrink, W., Nascimento, E., 1996. Histopathology and immunocytochemical study of type 3 and type 4 complement receptors in the liver and spleen of dogs naturally and experimentally infected with Leishmania (Leishmania) chagasi. Rev. Inst. Med. Trop. São Paulo 38, 81-89.

Tryphonas, L., Zawidzka, Z., Bernard, M.A., Jansen, E.A., 1997. Visceral leishmaniasis in a dog: clinical, hematological and pathological observations. Can. J. Comp. Méd. 41, 1-12. 